\title{
Grain size effect on the R-phase transformation of nanocrystalline NiTi shape memory alloys
}

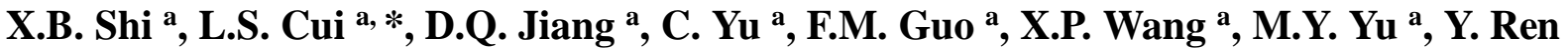 \\ c, Y. Liu ${ }^{\text {b }}$ \\ a State Key Laboratory of Heavy Oil Processing, China University of Petroleum, Beijing, \\ Changping 102249, China \\ b School of Mechanical and Chemical Engineering, The University of Western Australia, \\ Crawley, WA 6009, Australia \\ c X-ray Science Division, Argonne National Laboratory, Argonne, IL 60439, USA. \\ *Corresponding author. Tel.: +86 10 89731158; fax: +86 10 89731158; e-mail: \\ lishancui63@126.com.
}

\begin{abstract}
This study investigated the effect of nanograins size on the R-phase transformation of a nanocrystalline Ti-50.2at\%Ni alloy. The nanometric grain size was created by severe cold deformation and low temperature anneal. It was found that in the recrystallized state, nanograin sizes $(<100 \mathrm{~nm})$ was effective in suppressing the $\mathrm{B} 2 \rightarrow \mathrm{B} 19$ ' martensitic transformation and revealing the $\mathrm{B} 2 \leftrightarrow \mathrm{R}$ transformation. The $\mathrm{B} 2 \leftrightarrow \mathrm{R}$ transformation temperature was found to increase with decreasing grain size within the range of 22-155 nm.
\end{abstract}

Keywords:

A. Intermetallics; B. Phase transformation; C. Nanocrystals; D. Microstructure; F. Electron microscopy, transmission

\section{Introduction}

It is known that near-equiatomic NiTi shape memory alloys (SMAs) exhibit three martensitic transformations, between a high temperature B2 phase and a trigonal phase, conventionally known as the R-phase (B2↔R), between the B2 phase and a monoclinic B19' phase, known as the martensite (B2 $\leftrightarrow$ B19'), and the $\mathrm{R} \leftrightarrow \mathrm{B} 19^{\prime}$ ' phase transformation. The $\mathrm{B} 2 \leftrightarrow \mathrm{R}$ transformation is characteristic of a small transformation strain $(\sim 1 \%)$ and small thermal hysteresis $(<5 \mathrm{~K}$ ), as compared to the $\mathrm{B} 2 \leftrightarrow \mathrm{B} 19$ ' martensitic transformation. The $\mathrm{B} 2 \leftrightarrow \mathrm{R}$ transformation does not occur in defect-free binary NiTi alloys, but can be introduced by several means, including addition of a third element (e.g., Fe, Al [1,2]), precipitation aging [3] and introduction of dislocations (e.g., by cold working [4] and thermal cycling [5]). Previous studies have shown that the R-phase transformation temperature is much less sensitive to metallurgical conditions [3,6,7] compared to the $\mathrm{B} 2 \leftrightarrow \mathrm{B} 19$ ' transformation, apparently due to its relatively smaller lattice distortion. Whereas being an advantage for some applications, the more stable transformation temperature also implies limited ability to 
be adjusted to suit a particular application condition. One way to alter the R-phase temperature is aging for Ni-rich NiTi alloys [6,7]. The reason is that the formation of $\mathrm{Ti}_{3} \mathrm{Ni}_{4}$ precipitates reduces the Ni content of the NiTi matrix, and a lower Ni content (closer to the B2 stoichiometry) contributes to a higher R-phase transformation temperature in NiTi alloys. Cold deformation can reduce the temperature of the R-phase transformation by forming high-density defects [8]. A study by Chang et al. reported the effect of grain size on the R-phase transformation temperature, showing a lowered R-phase transformation temperature with reduced grain sizes in the surface regions of a cold rolled and annealed plate of a Ti-50at\%Ni alloy compared to the center of the plate where the grain size is larger [9]. The authors attributed the effect to the influence of the relatively higher density of dislocations and higher volume fraction of grain boundaries in the surface regions, which suppressed the $\mathrm{R}$-phase transformation. This hypothesis is consistent with the expectation of their effects on suppressing the martensitic transformation temperature. Tsuchiya et al. [16] reported a study of the effect of severe high pressure torsion on the mechanical and transformation behaviour of NiTi. In this work they observed that the B2 $\rightarrow \mathrm{R}$ transformation temperature increased with decreasing ageing temperature after the severe deformation, suggesting a grain size effect.

Development of nanoscale actuators and sensors in recent years calls for functional materials with small dimensions and high strengths. High strength nanocrystalline NiTi alloys which experience the R-phase transformation with a small thermal hysteresis are ideal candidates for these applications. Nanocrystalline metals often experience drastically different transformation characteristics compared to their coarse-grained forms [10-19]. To facilitate the application of R-phase transformation in nanocrystalline $\mathrm{NiTi}$ alloys, it is necessary to characterize the effect of grain size on transformation behaviour. In this study, the grain size effect on the R-phase transformation temperature of a nanocrystalline Ti-50.2at\%Ni alloy was investigated.

\section{Experimental details}

A Ti-50.2at\%Ni alloy wire provided by the General Research Institute for Non-Ferrous Metals, China was used in this study. The wire was $0.82 \mathrm{~mm}$ in diameter in cold-drawn state. The wire was annealed at $750{ }^{\circ} \mathrm{C}$ for $90 \mathrm{~s}$ in air followed by air cooling and then cold drawn into a diameter of $0.43 \mathrm{~mm}$, effecting an area reduction of $72.5 \%$. Samples of the cold-drawn wire were annealed at $350,400,450$ and $500{ }^{\circ} \mathrm{C}$ for $0.6 \mathrm{ks}$. Small specimens were cut from the annealed wire using a diamond cut-off wheel and analyzed using a Netzsch DSC 204 F1 Phoenix differential scanning calorimeter (DSC) with a heating/cooling rate of $10{ }^{\circ} \mathrm{C} / \mathrm{min}$. Samples used for microstructure observation were mechanically polished and then ion milled using a Gatan 691 PIPS instrument. Transmission electron microscopic (TEM) observation was carried out using a FEI tecnai F20 (operating at $200 \mathrm{kV}$ ) equipped with a Gatan slow scan CCD camera. The average grain sizes were acquired by measuring 200 individual grains from the TEM micrographs manually using the Digital Micrograph software installed with the Gatan CCD camera. High-energy X-ray (HEX) diffraction measurements were performed at the 11-ID-C beamline of the advanced photon source at Argonne National Laboratory, 
USA.

\section{Results}

Fig. 1 shows TEM micrographs revealing the microstructures of the NiTi wire before and after annealing. Fig. 1(a) is a bright field image and a corresponding selected-area electron diffraction (SAED) pattern of the cold-drawn sample. The arrow indicates the drawing direction of the sample. The cold-drawn NiTi wire contained nanograins embedded in an amorphous NiTi matrix. Fig. 1(b) is a bright field TEM image of a sample annealed at $350{ }^{\circ} \mathrm{C}$ and its SAED pattern. The microstructure is completely nanocrystalline and the SAED pattern reveals no diffused ring, indicating that the amorphous phase has been fully crystallized after annealing. Fig. 1(c) shows grain size distribution for the sample annealed at $350{ }^{\circ} \mathrm{C}$. The majority ( 84\%) grains are smaller than $15 \mathrm{~nm}$, with an average grain size of 10 $\mathrm{nm}$ based on measurement of 200 grains. Fig. 1(d)-(f) show bright field TEM images and SAED patterns of the samples annealed at 400,450 and $500{ }^{\circ} \mathrm{C}$, respectively. The average grain sizes of these samples are determined to be 22, 56 and $155 \mathrm{~nm}$, respectively. Close examination was carried out by TEM observation of 30 grains randomly for each of the samples. The bright-field TEM and SAED analysis reveal no precipitates and few dislocations.
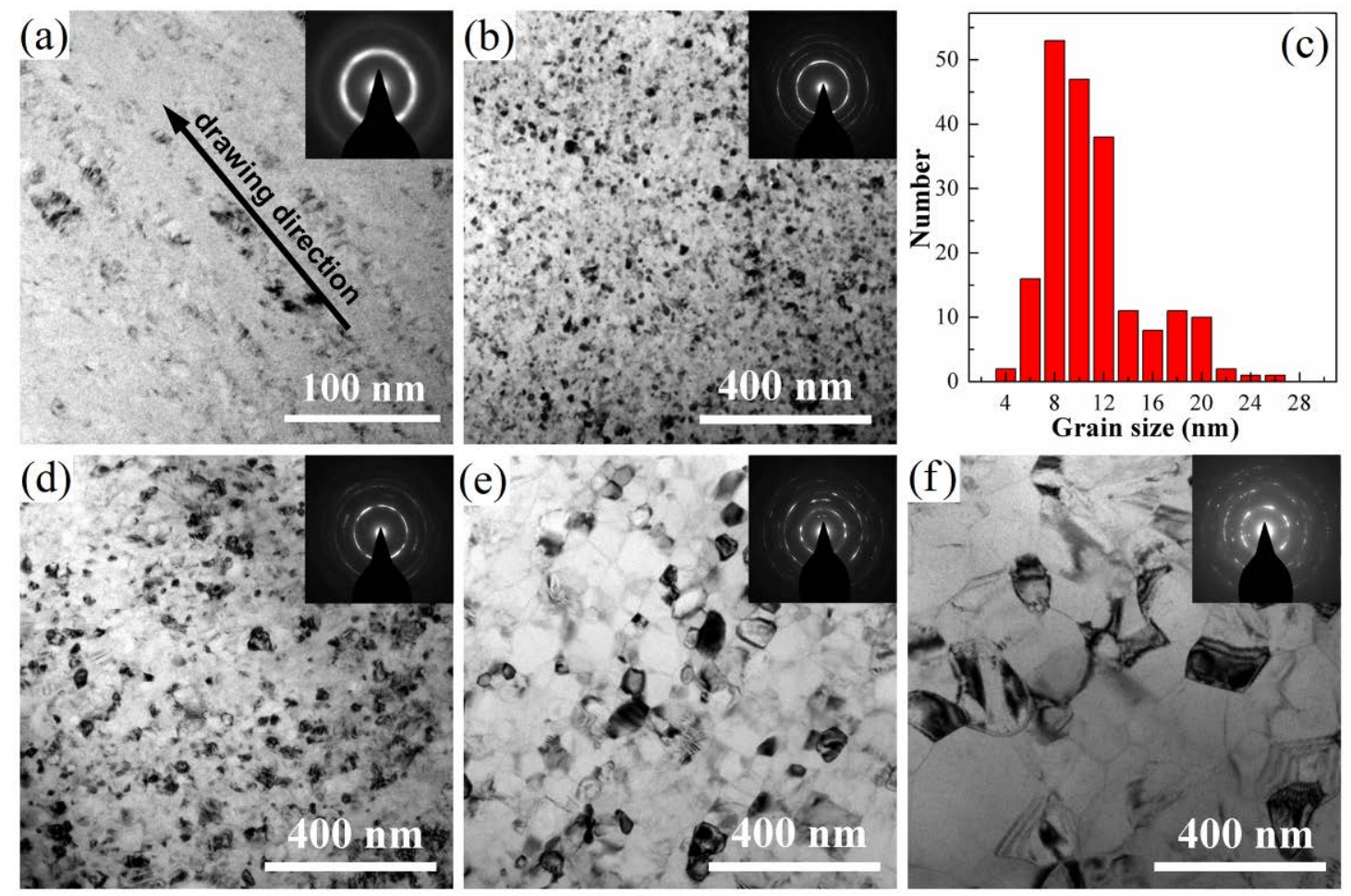

Fig. 1. TEM bright-field micrographs and selected-area electron diffraction patterns of the Ti-50.2at\%Ni wire: (a) before annealing, (b) annealed at $350{ }^{\circ} \mathrm{C}$; (c) grain size distribution of the sample annealed at $350{ }^{\circ} \mathrm{C}$; (d) annealed at $400{ }^{\circ} \mathrm{C}$; (e) annealed at $450{ }^{\circ} \mathrm{C}$; (f) annealed at $500^{\circ} \mathrm{C}$.

Fig. 2(a) shows DSC analysis of the transformation behaviors of the annealed 
nanocrystalline Ti-50.2at\%Ni wires. It is seen that for most samples only the $\mathrm{B} 2 \leftrightarrow \mathrm{R}$ transformation was detected within the temperature range tested, except the sample with 155 $\mathrm{nm}$ of average grain size (the largest). Fig. 2(b) shows the influence of grain size on the $\mathrm{B} 2 \leftrightarrow \mathrm{R}$ transformation peak temperatures. The $\mathrm{B} 2 \leftrightarrow \mathrm{R}$ transformation temperatures increased continuously with decreasing grain size to $22 \mathrm{~nm}$. The sample with the average grain size of $10 \mathrm{~nm}$ has almost the same transformation temperature to the sample with the grain size of 22 $\mathrm{nm}$, whereas the transformation exothermic peak area is much smaller. This size effect is different from those of the coarse-grained NiTi alloys, in which the R-phase transformation temperature was found to decrease with decreasing grain size [9]. DSC analysis in Fig. 2c shows that the crystallization temperature is $\sim 350^{\circ} \mathrm{C}$ and all samples presented in this work were annealed at above the crystallization temperature. TEM examination confirms that all samples were fully crystallized after annealing. Fig. 2d shows the one-dimensional high energy X-ray (HEX) diffraction spectra of the 350 and $500{ }^{\circ} \mathrm{C}$ annealed samples. It reveals no $\mathrm{Ti}_{3} \mathrm{Ni}_{4}$ precipitate diffraction peaks.
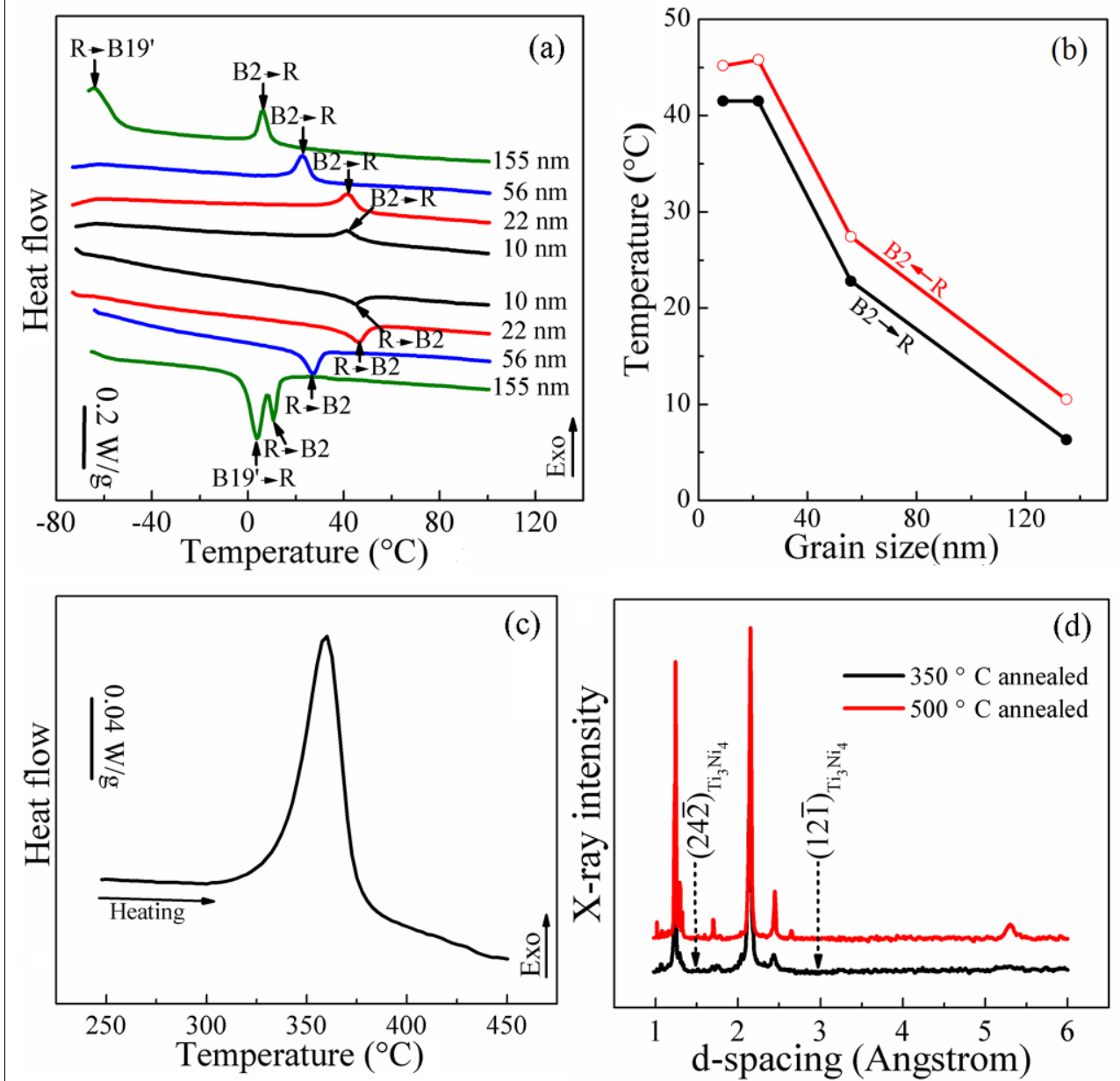

Fig. 2. The transformation behavior of nanocrystalline Ti-50.2at\%Ni wires with different grain sizes: (a) DSC measurements of the samples; (b) effect of grain size on the peak temperatures of the R-phase transformation; (c) DSC curve of the crystallization of cold-drawn sample upon heating; (d) high energy X-ray diffraction spectra of $350{ }^{\circ} \mathrm{C}$ and $500{ }^{\circ} \mathrm{C}$ annealed samples. The two arrows mark out d-spacing 
positions of two major diffraction peaks of $\mathrm{Ti}_{3} \mathrm{Ni}_{4}$.

Fig. 3 shows HRTEM analysis of a nanograin of about $30 \mathrm{~nm}$ in size in the sample annealed at $400{ }^{\circ} \mathrm{C}$. Fig. 3(a) is a HRTEM image of the nanograin with a beam direction parallel to $[111]_{\mathrm{B} 2}$. The grain is divided into two regions. Region A has a fast-Fourier transform (FFT) pattern shown as in Fig. 3(b), and the pattern of region B is shown in Fig. 3(c). The FFT pattern of region A shows a typical B2 parent phase structure in [111 $]_{\mathrm{B} 2}$ direction. In contrast, the FFT pattern of region B shows clearly some low intensity spots at $1 / 3$ positions in between the fundamental spots of the B2 phase, as indicated in the figure. These weak spots correspond to the R-phase. Fig.3 (d) shows the FFT pattern of the whole grain and Fig. 3(e) shows the inverse FFT corresponding to the R-phase spots masked in Fig.3 (d). The interface was distinguished from the inverse FFT image (Fig. 3(e)) in which R-phase region shows inerratic stripes clearly. It was observed in HRTEM analysis that areas of region $\mathrm{B}$ are all close to grain boundaries. This observation implies that grain boundaries encourage and stabilize the R-phase. The HRTEM analysis of a grain about $20 \mathrm{~nm}$ in diameter and the corresponding inverse FFT image, shown as Fig. 3(f) and (g), reveal that almost the whole grain shows inerratic stripes which corresponding to R-phase spots. This result consists to the result of Fig. 2 that R-phase was stabilized in smaller grains.
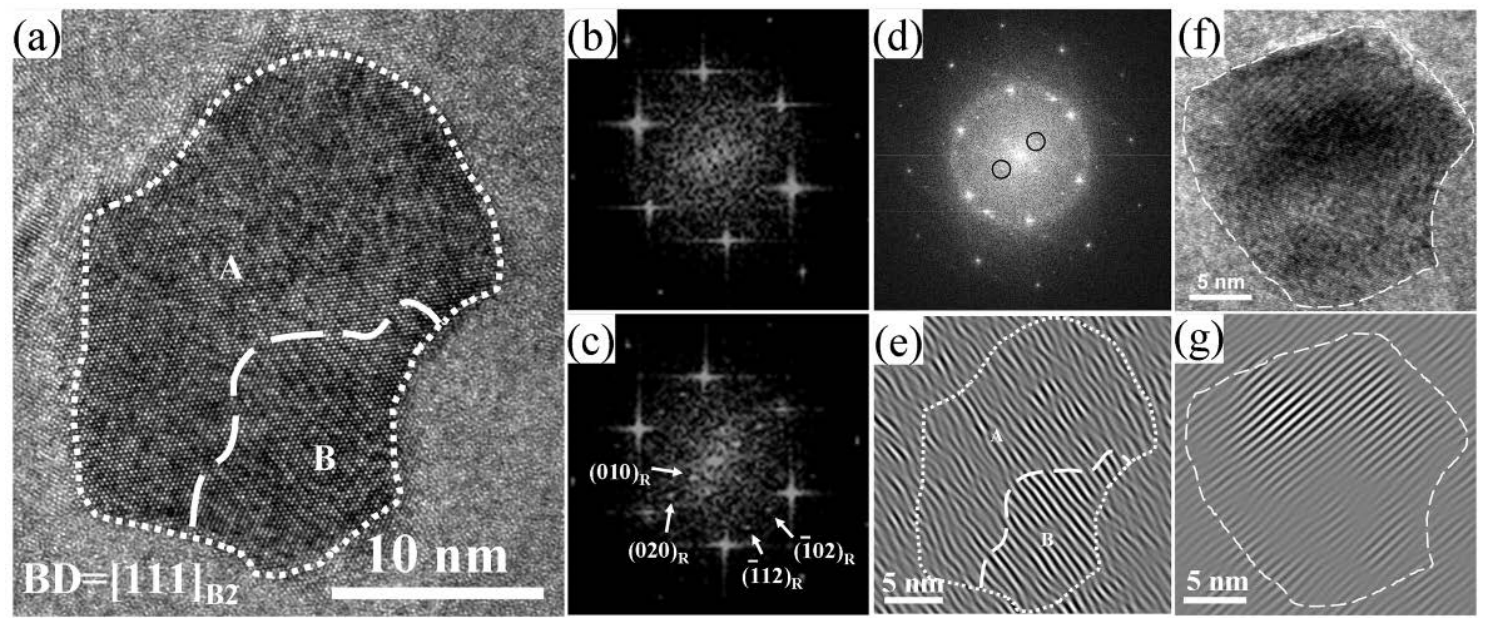

Fig. 3. HRTEM analysis of two nanograins about $30 \mathrm{~nm}$ and $20 \mathrm{~nm}$ in diameter: (a) HRTEM image of the nanograin about $30 \mathrm{~nm}$ in diameter; (b, c) fast Fourier transform image of the central area (region A) and the grain-boundary area (region B) of the nanograin shown in (a), respectively; (d) fast Fourier transform image of the whole grain in (a); (e) inverse FFT image corresponding to the FFT spots masked in (d); (f) HRTEM image of the nanograin about $20 \mathrm{~nm}$ in diameter; (g) inverse FFT image of (f) corresponding to the R-phase spot in FFT image.

\section{Discussion}

4.1 Exhibiting of R-phase transformation

The occurrence of the $\mathrm{B} 2 \rightarrow \mathrm{R}$ transformation in the recrystallized nanocrystalline 
samples, as evidenced in Fig. 2(a), is worth noting. It is known that fully annealed, precipitate-free binary near-equiatomic NiTi alloys do not exhibit the $\mathrm{B} 2 \rightarrow \mathrm{R}$ transformation and that the $\mathrm{B} 2 \rightarrow \mathrm{R}$ transformation can be induced by cold working [4], thermal cycling [5] or precipitation ageing [3]. The occurrence of the $\mathrm{B} 2 \rightarrow \mathrm{R}$ transformation in all the samples used in this study demonstrates that the nanometric grain size is another factor promoting the $\mathrm{B} 2 \rightarrow \mathrm{R}$ transformation. This is attributed to the effect of suppressing the martensitic transformation, as confirmed by several studies [10,11,17-19], revealing the $B 2 \rightarrow R$ transformation. The suppression of the $\mathrm{B} 2 \rightarrow \mathrm{B} 19^{\prime}$ in nanograins is believed to be due to the limited space to contain enough number of martensite variants to form effective self-accommodation structures for the accommodation of the large lattice distortion of the martensite [10,11]. This is consistent with the observation that B19' martensite is only observed in the sample with the largest grain size $(155 \mathrm{~nm})$, at a very low temperature.

\subsection{Grain size effect on R-phase transformation}

It is also evident in Fig. 2 that the R-phase transformation temperature has a clear dependence on the grain size (in the range observed), i.e., the $\mathrm{R}$ phase temperature increases with decreasing grain size. Despite the general belief, there is very limited experimental evidence of the effect of grain size on the R-phase transformation temperature in the literature. Chang et al reported that the R-phase temperature is lower in the surface regions of cold-rolled and then annealed Ti-50at\%Ni plate, where the grain size is small, than that in the center of the plate, where the grain size is large [9], implying an effect opposite to what is reported here. Whereas unclear about the reasons of this difference, the grain sizes in Chang's work are in the range of 7-72 $\mu \mathrm{m}$ whereas those in this study are 10-155 nm, 2 3 orders of magnitude different. Another piece of direct evidence is provided by Waitz et al [11]. In their investigation on the effect of grain size on the B2 $\rightarrow$ B19' martensitic transformation via TEM examination, they observed the appearance of the R-phase in regions of small (15 150 nm) grains whereas in regions of large grain sizes (>150 nm) only B19' martensite is observed. This observation is consistent with the findings of this study. Summarizing the evidences reported in the literature, the results presented here appear to be the more considered correlation between nanograin size and R-phase transformation temperature through careful design of experiment to allow the elimination of the side effects of dislocations due to incomplete crystallization or precipitates caused by ageing.

It is known [6-8] that the temperature of the B2 $\rightarrow$ R transformation can be affected by formation of precipitates and dislocations, generally to lower the transformation temperature by resisting the lattice distortion of the transformation or to increase the transformation temperature by reducing the Ni content of the matrix. The SAED patterns shown in Fig. 1 indicate that the annealed samples are practically precipitate-free. The TEM observation shows that the nanograin contains few dislocations (Fig. 1). In this regard, precipitates and dislocations cannot the primary factors contributing to the increase of the R-phase transformation temperature with reduction of grain size observed in this study. Another possible mechanism suggested in the literature is that grain boundary regions promote heterogeneous nucleation [10] and thus raise the temperature of the R-phase formation. As 
per this hypothesis, a broad transformation peak corresponding to the transition in Gibbs free energy state between grain boundary and grain interior regions will be expected and changes in grain size will result in changes in the relative volume fractions of the two regions, thus the shape of the peak, but not the start transformation temperature which corresponding to the nucleation at grain boundary. This expectation clearly does not agree with the evidence presented in Fig. 2(a). Peterlechner et al. [16] tried to explain the grain size effect within a thermodynamic framework, and predicted that $\mathrm{B} 2 \rightarrow \mathrm{R}$ transformation may be suppressed by an increase of elastic strain energy, which may be caused by a reduction in grain size. However, our results disagree with this prediction. Considering the above, a credible explanation to the nanograin size dependence of the R-phase transformation temperature is yet to be established.

\section{Conclusions}

This study has investigated the grain size effect on R-phase transformation of a nanocrystalline Ti-50.2at\%Ni alloy. The results show that:

(1) In the nanocrystalline NiTi alloy, nanograin sizes $(<100 \mathrm{~nm})$ was effective in suppressing the B2 $\rightarrow$ B19' martensitic transformation and revealing the B2↔R transformation.

(2) The $B 2 \leftrightarrow R$ transformation temperature was found to increase with decreasing grain size within the range of $22-155 \mathrm{~nm}$. This size effect is independent from the formation of precipitates and dislocations.

\section{Acknowledgment}

This work was supported by the key program project of National Natural Science Foundation of China (NSFC) (51231008), the National 973 programs of China (2012CB619400), the NSFC (51001119) and the Key Project of Chinese Ministry of Education (313055). The use of the Advanced Photon Source was supported by the US Department of Energy, Office of Science, and Office of Basic Energy Science, Office of Basic Energy Sciences, under Contract No. DE-AC02-06CH11357.

\section{Reference}

[1] Hwang C, Meichle M, Salamon M, Wayman C. Transformation behaviour of a Ti50Ni47Fe3 alloy I. Premartensitic phenomena and the incommensurate. Phase. Phil Mag A 47 (1983) 9-30.

[2] Hwang C, Wayman C. Microstructural Features of Charge Density Wave (CDW) and Martensitic Transformations in TiNiFe and TiNiAl Alloys. Scripta Metall 17 (1983) 1449.

[3] Allafi J, Ren X, Eggeler G. The mechanism of multistage martensitic transformations in aged Ni-rich NiTi shape memory alloys Acta Mater 50 (2002) 793-803.

[4] Lin $\mathrm{H}$, Wu S, Chou T, Kao $\mathrm{H}$. The effects of cold rolling on the martensitic transformation of an equiatomic TiNi alloy. Acta Metall. Mater. 39 (1991) 2069-2080.

[5] Mccormick P, Liu Y. Thermodynamic analysis of the martensitic transformation in 
NiTi-II. Effect of transformation cycling. Acta Metal. Mater. 42 (1994) 2407-2413.

[6] Kim J, Liu Y, Miyazaki S. Ageing-induced two-stage R-phase transformation in Ti-50.9at.\%Ni. Acta Mater. 52 (2004) 487-449.

[7] Zhou Y, Zhang J, Fan G, Ding X, Sun J, Ren X, Otsuka K. Origin of 2-stage R-phase transformation in low-temperature aged Ni-rich Ti-Ni alloys. Acta Mater. 53 (2005) 5365-5377.

[8] Chrobak D, Stróż D. Two-stage R phase transformation in a cold-rolled and annealed Ti-50.6 at.\%Ni alloy. Scripta Mater. 52 (2005) 757-760.

[9] Chang S, Wu S, Chang G. Grain size effect on multiple-stage transformations of a cold-rolled and annealed equiatomic TiNi alloy. Scripta Mater. 52 (2005) 1341-1346.

[10] Waitz T, Karnthaler H. Martensitic transformation of NiTi nanocrystals embedded in an amorphous matrix. Acta Mater. 52 (2004) 5461-5469.

[11] Waitz T, Kazykhanov V, Karnthaler H. Martensitic phase transformations in nanocrystalline NiTi studied by TEM. Acta Mater. 52 (2004) 137-147.

[12] Xu W, Song X, Lu N, Huang C. Thermodynamic and experimental study on phase stability in nanocrystalline alloys. Acta Mater. 58 (2010) 396-407.

[13] Meng Q, Zhou N, Rong Y, Chen S, Hsu T. Size effect on the Fe nanocrystalline phase transformation. Acta Mater. 50 (2002) 4563-4570.

[14] Mao S, Li H, Liu Y, Deng Q, Wang L, Zhang Y, Zhang Z, Han X. Stress-induced martensitic transformation in nanometric NiTi shape memory alloy strips: An in situ TEM study of the thickness/size effect. Journal of Alloys and Compounds 579 (2013) 100-111.

[15] Tsuchiya K, Ohnuma M, Nakajima K, Koike T, Hada Y, Todaka Y and Umemoto M. Microstructures and enhanced properties of SPD-processed TiNi shape memory alloy. Mater. Res. Soc. Symp. Proc 1129 (2009), 113-124.

[16] Peterlechner M, Waitz T, Gammer C, and Antretter T. Martensitic phase transformations of nanocrystalline NiTi shape memory alloys processed by repeated cold rolling. International Journal of Materials Research 102 (2011) 634-642.

[17] Jiang S, Zhang Y, Zhao L, Zheng Y. Influence of annealing on NiTi shape memory alloy subjected to severe plastic deformation. Intermetallics 32 (2013) 344-351.

[18] Waitz T; Antretter T; Fischer F; Karnthaler H. Size effects on martensitic phase transformations in nanocrystalline NiTi shape memory alloys. Materials Science and Technology, 24 (2008) 934-940.

[19] Waitz T, Antretter T, Fischer F, Simha N, Karnthaler H. Size effects on the martensitic phase transformation of NiTi nanograins. Journal of the Mechanics and Physics of Solids 55 (2007) 419-444. 